\title{
Cardiac Motions Classification on Sequential PSAX Echocardiogram
}

\author{
Adam Shidqul Aziz ${ }^{1}$, Riyanto Sigit ${ }^{2}$, Achmad Basuki ${ }^{3}$, Taufik Hidayat ${ }^{4}$ \\ 1,2,3 Informatics and Computer Engineering, Politeknik Elektronika Negeri Surabaya, Surabaya \\ ${ }^{4}$ Department of Pediatrics, Airlangga University, Surabaya
}

\begin{tabular}{l} 
Article Info \\
\hline Article history: \\
Received Jul 1, 2018 \\
Revised Aug 30, 2018 \\
Accepted Sep 21, 2018 \\
\hline Keywords: \\
Euclidean distance \\
Flow determination algorithm \\
Lucas-Kanade optical flow \\
Neural network \\
Semi-automatically cardiac \\
motions classification
\end{tabular}

Article Info motions classification

\begin{abstract}
Cardiac wall motions classification on 2-dimensional (2D) echocardiographic images is an important issue for quantitative diagnosiing of heart disease. Unfortunately, the bad quality of echocardiogram cause computationally classification on cardiac wall motions is still become a big homework for many researchers to provide the best result. Echocardiogram is produced by soundwaves which absolutely make its images have speckle noise in different intensity. Therefore, this research improves a set of methodology to classify cardiac wall motion semi-automatically. Raw echocardiogram will be enhanced and segmented to take the boundary of endocardium of left ventricular in PSAX cardiac images. New improvement of Semiautomatically methodology is approach on detecting the contour of endocardium and will be inputed as good features in Lucas-Kanade Optical Flow in all sequential echocargraphic images. On classifying cardiac wall motions, this research proposes two important features including length of displacement and flow direction. New proprosed flow determination algorithm and Euclidean distance is used to calculate those features. All the features will be trained by Neural Network (NN) and validated by Leave One Out (LOO) to get accurate result. NN method, which is validated by LOO, has the best result of $81.82 \%$ correctness than the other compared methods.
\end{abstract}

Copyright $@ 2018$ Institute of Advanced Engineering and Science. All rights reserved.

\section{Corresponding Author:}

Adam Shidqul Aziz,

Informatics and Computer Engineering,

Politeknik Elektronika Negeri Surabaya, Surabaya.

Email: aziz.addam@gmail.com

\section{INTRODUCTION}

Heart, which is located within thoracic cavity, is one of the most important organ in the body. The size of typical heart is just like a human fist: $12 \mathrm{~cm}$ in length, $8 \mathrm{~cm}$ in wide, and $6 \mathrm{~cm}$ in thickness. However, heart has a vital job of pumping and spreading blood throughout the body [1]. heart flows oxygen-containing blood throughout the body. Oxygen will decompose glucose in the blood and produce Adenosine Triphosphate (ATP) and become a useful energy for cellular respiration. The death statistic report of the cardiovascular disease in 2018 has been alarming. World Health Organization (WHO) reported that cardiovascular disease caused 17,7 million deaths every year, it means $31 \%$ of all global deaths are caused by cardiovascular disease [2]. Therefore, echocardiography appears as one of non-invasive and painless technology to create image of human heart. The expert in echocardiography diagnose heart condition based on some symptoms which appear in the image. One example of symptom is cardiac wall motion. Cardiac wall motion can give an indication whether the heart is healthy or not. Nevertheless, echocardiography has some limitations including image quality, operator dependency, and interpreter dependency [3]. Those limitation affects an impact on the accuracy of the doctor's diagnosis. The accuracy of the doctor's diagnosis depends on the doctor's knowledge and experience. This case motivates some of engineers start to create various inventions related to image processing on echocardiogram. Some of the inventions focus on 
enhancement [4-7]. The objective of enhancement is to remove speckle noise and sharpen in echocardiographic image. Most of medical research propose median filter to remove speckle noise in image. The other inventions focus on classifying the echocardiogram become some of specific condition [8-9]. Current research, not only 2-dimensional echocardiogram, but also 3-dimensional echocardiographic image. So, the invention on diagnosing echocardiographic image is still opened.

The high risk of mortality that caused by cardiovascular disease motivates researchers to develop a computational diagnosis with their own approach in many types of resource. Especially on 2D or 3D echocardiographic image, this type of resource continues to be a trend for researcher to diagnose. Meysam Siyah Mansoory, et.al [9] evaluated heart movement to diagnose heart disease. He used the Independent Computer Algorithm (ICA) to obtain the features of echocardiography. Meysam used the neural network to classify and obtained the highest accuracy of $95.7 \%$ for normal data and $93.8 \%$ for abnormal data. Sarina Mansor and J Alison Noble [10] in 2008 conducted a classification study on wall movement of echocardiographic images using the Hidden Markov Model (HMM) method and using Leave One Out Validation (LOO) method resulted in an average accuracy of $80 \%$.

In this research, we would like to investigate whether the flow direction and length of movement as features on cardiac wall motion can classify heart condition. Those features are the technique of the echocardiography expert to diagnose cardiac wall motion. We used semi automatically good feature for Lucas-Kanade Optical Flow to obtain those features. Finally, artificial neural network is performed to classify the cardiac wall motion.

\section{WALL MOTION ABNORMALITIES}

Echocardiography produces four types of views: short axis (SAX), long axis (LAX), apical two chamber (A2C), apical four chamber (A4C). nevertheless, this research focus on SAX view. On PSAX echocardiogram, the expert will observe wall motions of left ventricular to determine the current first judgement of heart condition. abnormalities of heart will be indicated by motions of endocardium and pericardium tissue. Heart movement and size of the heart segment wall when contracting and relaxation have an indication of heart disease. Pandian et al [11] explains that movement of the left ventricular wall that is not symmetry and thickening of the heart wall in general gives a sign of myocardial ischemia or infarction. He concluded that the thickening and movement of the heart wall provides an indication of the heart's wall abnormalities.

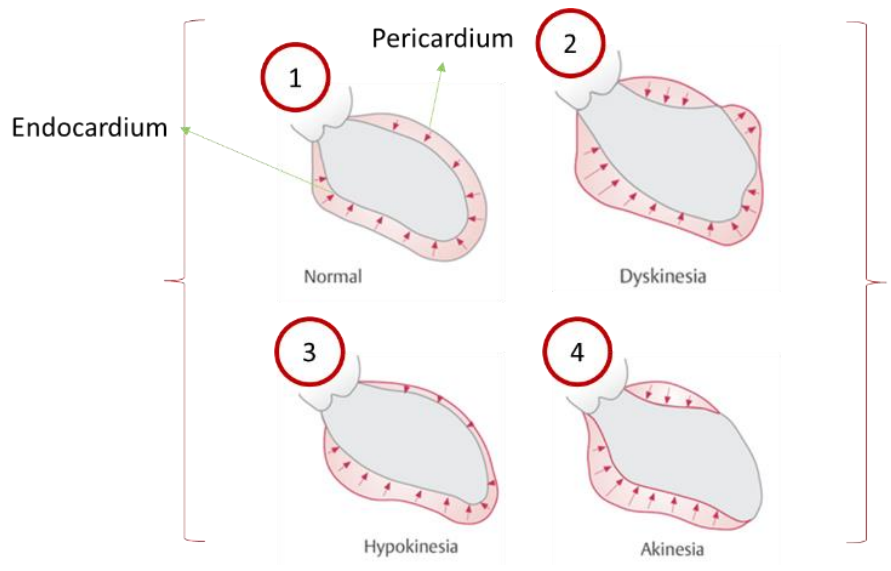

Figure 1. Types of Wall Motions Abnormalities

Figure 1 shows there are four types of wall motions abnormalities such as normal, dyskinesia, hypokinesia, akinesia. Like explained by Catherine Otto [12], normal condition is endocardium moving into deep toward the center normally during systole, dyskinesia is endocardium moving in different directions, hypokinesia is endocardium moving into deep toward the center slowly during systole (amplitude less than 5 $\mathrm{mm}$ ), and akinesia is endocardium not moving at all. In this research, Dyskinesia, hypokinesia, and akinesia will be classified as abnormal heart condition. 


\section{RESEARCH METHOD}

Main idea in this research is how to classify cardiac motions on echocardiographic images by using flow direction and length of movement as features. Boundary of endocardium in this research is obtained semi-automatically. It means that system just need a center point coordinate of endocardium in first running.

This research using set of various methods before getting final diagnose of wall motion. Figure 2 shows there are four steps in the proposed algorithm including enhancement, segmentation, feature extraction, and classification. Enhancement and segmentation has been completely explained by Riyanto Sigit et.al [13] in his paper.

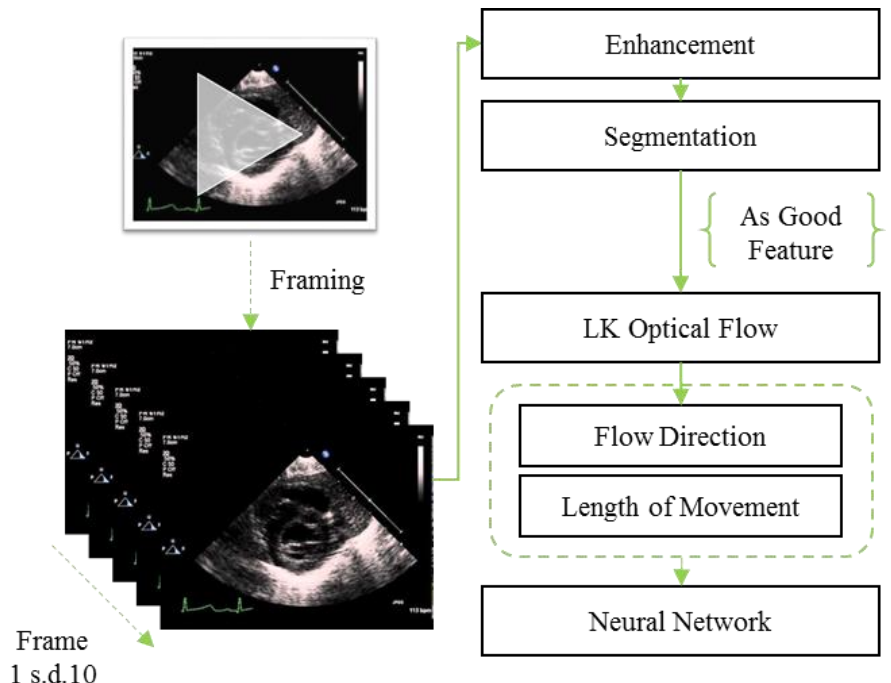

Figure 2. Proposed Algorithm

\subsection{Enhancement}

Speckle noise is a main problem in the most of echocardiographic image. The higher intensity of speckle noise, the more difficult to be observed for the doctor. So, enhancement is needed to remove speckle noise and the final result to convert object of image into edge. Enhancement is divided into four steps. The explanation is as follows:

\section{a) Median High Boost Filter}

Median High Boost filter is a new modified of band pass filter. The high-boost filter can be used to enhance high frequency component while still keeping the low frequency components. Usually, High Boost filter uses mean fitler as low pass filter in its combination. However, this research uses median filter as low pass filter in composing High Boost Filter. Median filter has good performance on reducing speckle noise in image. The fundamental equation of high boost filter is mentioned as follows:

$$
I_{h b}=I_{0}+c * I_{h p}
$$

Equation 1 explains that high boost image (Ihb) is resulted by appending pixel value of original image (I0) with high pass image (Ihp) and multipled with determined constanta (c).

$$
W_{h b}=W_{a p}+c * W_{h p}
$$

Equation 2 describes the high boost convolution kernel. Kernel is obtained by appeding all pass kernel (Wap) and contanta (c) multipled by high pass kernel (Whp).

$$
I_{h p}=I_{0}-I_{l p}
$$

Otherside, high pass image (Ihp) can be produced by original image (I0) minus low pass image (Ilp). Low pass filter is used to remove or reduce noise. In this research, high boost filter uses median filter to remove speckle noise containing in echocardiogram. 


\section{b) Morphology}

Morphology is a broad set of image processing operations that process images based on shapes. Morphological is divided into dilation and erosion. Combination of dilation and erosion makes two new algorithms of opening and closing.

$$
\begin{aligned}
& A \circ B=(A \ominus B) \oplus B \\
& A \circ B=(A \oplus B) \ominus B
\end{aligned}
$$

Equation 4 is an opening morphological. Opening is the dilation of the erosion of a set $\mathrm{A}$ by a structuring element B. Equation 5 is a closing morphological. Closing is a set (binary image) A by a structuring element $\mathrm{B}$ is the erosion of the dilation of that set.

\section{c) Thresholding}

Thresholding processing will convert image into binary image. This method will create a black white image. To convert image to binary, image must be convert into grayscale image and converti it into binary with determined threshold value. The formula of binary pixel determination is explained as follows:

$$
p(x, y)=\left\{\begin{array}{l}
0, p(x, y)<t h \\
1, p(x, y) \geq t h
\end{array}\right.
$$

\section{d) Canny Filter}

Canny Filter is an edge detection operator that uses al multi-stage algorithm to detect a wide range of edges in image. The Process of Canny edge detection algorithm can be broken down to 5 different steps:

1) Apply Gaussian filter to smooth the image in order to remove the noise

2) Find the intensity gradients of the image

3) Apply non-maximum suppression to get rid of spurious response to edge detection

4) Apply double threshold to determine potential edges

5) Track edge by hysteresis: Finalize the detection of edges by suppressing all the other edges that are weak and not connected to strong edges.

\subsection{Segmentation}

Segmentation is a next step after enhancement to take only the contour of endocardium. the contour will become good feature for Lucas-Kanade Optical Flow to estimate motion of endocardium in sequential echocardiographic images. There are three steps in segmentation method. The explanation is as follows:

\section{a) Region Filter}

Region Filter will remove all unnecessary contour and edge outside of determined range. Range value is determined manually from center point of endocardium to estimation of the outest of endocardium boundary.

\section{b) Collinear}

Collinear will remove contour and edge inside determined range. The equation of collinear described in Equation 7.

$$
x_{1}\left(y_{2}-y_{3}\right)+x_{2}\left(y_{3}-y_{1}\right)+x_{3}\left(y_{1}-y_{2}\right)=0
$$

Equation 7 explains about three points in one straight line. The third one will be detected as unnecessary point and will be removed.

\section{c) Triangle Equation}

This method is proposed specially by Riyanto Sigit et.al [13] to connect two unconnected line. Two determined points will be choosen based on the closest angle from those points. The explanation is as follows:

1) System has to determine the approximate center of the enclosed region that will be connected by calculating the centroid.

2) The endpoints of the boundary are marked as two couples point that will be connected. Those points can be illustrated as first point is B and the second one is $\mathrm{C}$. Then, the center point become the third point. So, BAC will be formed as triangle. 
3) The gap in the boundary is minimized by fixing one of the end points $\mathrm{m}$ such as point $\mathrm{B}$. point $\mathrm{C}$ is allowed to transverse inward along the boundary until the point at which the angle BAC is minimized is found.

4) The open boundary is then connected by a line from point $B$ to point $C$.

\subsection{Feature Extraction}

Lucas-Kanade Optical Flow is performed to estimate the boundary of endocardium in sequential echocardiographic images. Optical Flow uses intensity of image to determine identical pixel in another image. In Optical Flow $I(x, y, t)$ is the intensity of pixels with $(x, y)$ express location of the pixel and $\mathrm{t}$ express time. Basically, Optical Flow can be described by Equation 8 [3]:

$$
I(x, y, t)=I(x+\Delta x, y+\Delta y, t+\delta t)
$$

$\Delta x$ and $\Delta y$ are determined as below:

$$
\left\{\begin{array}{l}
\Delta x=u \delta t \\
\Delta y=u \delta t
\end{array}\right.
$$

$(u, v)$ are velocity components in horizontal and vertical directions at the point $(x, y) . \Delta x, \Delta y$ are movement at mentioned direction respectively. Moreover, $\delta \mathrm{t}$ is the small-time interval between two sequential frames.

This research splits video of echocardiogram become ten frames. All frames are a representation of one heart cycle from diastole to systole. By using Optical Flow, determining boundary of endocardium can performed in all sequential frames without repeating the initial process of enhancement and segmentation.
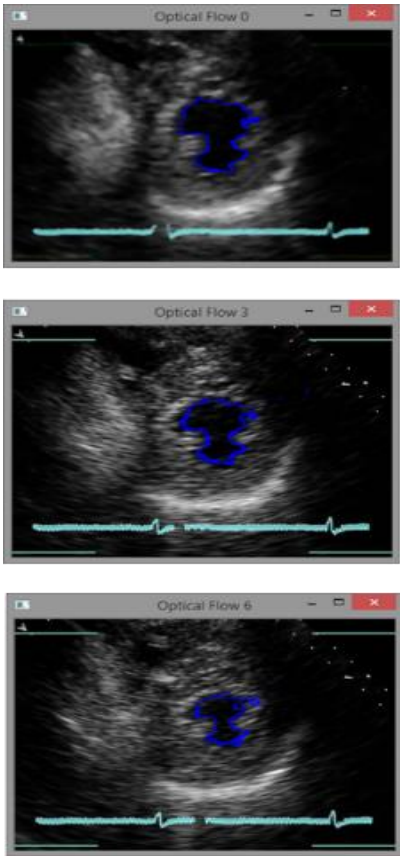
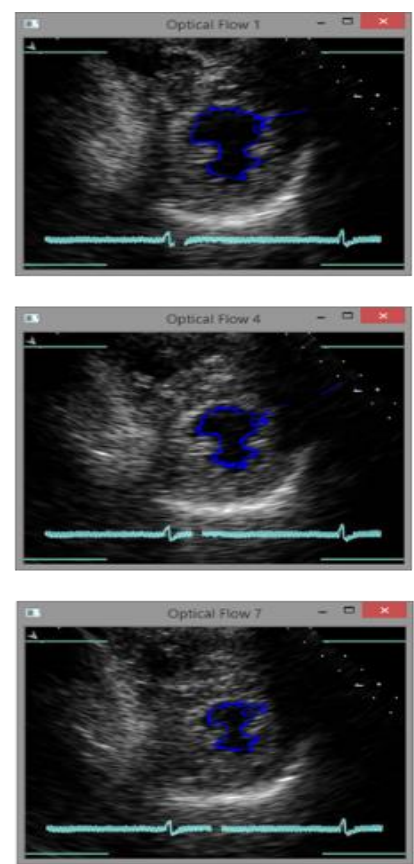
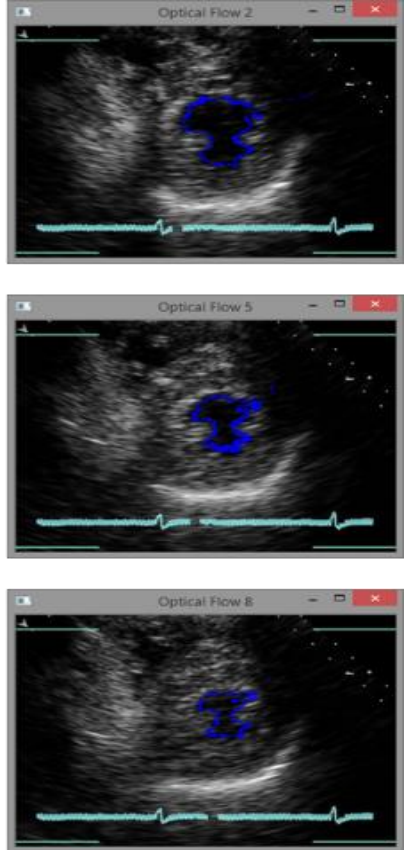

Figure 3. Lucas-Kanade Optical Flow Estimation

Boundary of endocardium that has been obtained from segmentation method will be good features in Optical Flow. Optical Flow of Lucas-Kanade will find identical pixel in next image based on the closest intensity in two pixels. Figure 3 shows Optical Flow estimation can find most of pixel between two images in one set sequential images. However, the success rate of Optical Flow estimation depends on the quality of all frames. If the next image has bad quality, then Optical Flow will be difficult to find identical pixel and surely many of next good features will be missed. This research uses 11 set of echocardiograms which are divided become two group class of normal and abnormal. Specifically, there are five normal and six abnormal echocardiograms. 
Every good features founded in every images will be extracted into necessary feature for classification. There are two features that is length of movement and flow of directions. Those features are basic symptom for the doctor to diagnose heart condition throughout wall motion in SAX echocardiogram.

\subsubsection{Length of Movement or Displacement}

Length of movement is a length between two points from image A to image B where image B is the next good feature image on sequential echocardiogram. Point in image B is good feature estimated by Optical Flow. The equation of length of displacement is described below:

$$
d=\sqrt{\left(x_{n+1}-x_{n}\right)^{2}+\left(y_{n+1}-y_{n}\right)^{2}}
$$
and $\mathrm{y}$.

Equation 10 explains difference $(\mathrm{d})$ is obtained from squared root of sum of squared difference of $\mathrm{x}$

\subsubsection{Flow Direction Determination}

Flow Direction is a feature that describes a direction of movement between to pixel. There are two kind of directions that is unchanged, inward and outward. Unchanged pixel is two pixels stay in one place. Inward is good features moving inside towards the center point of endocardium. Outward is good features moving outside toward from the center point of endocardium.

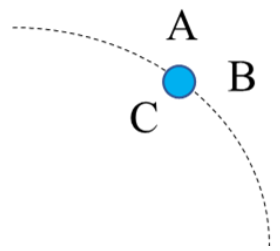

(1)

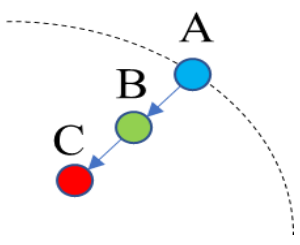

(4)

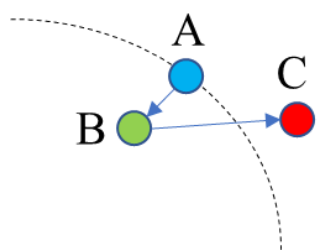

(2)

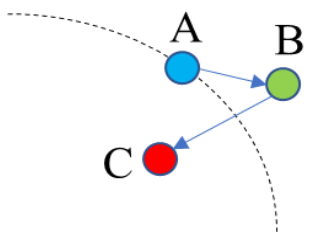

(5)

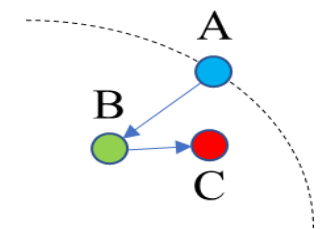

(3)

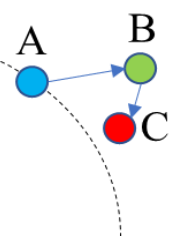

(6)

Figure 4. Algorithm of Determination of Flow Direction

Figure 4 shows some algorithms to determine kind of flow direction between two sequential images. flow direction determination uses first boundary of endocardium as an initial contour. There are six algorithms described below:

a) Image part (1): an unchanged pixel is a condition where the next estimated-pixel (C) does not change against previous pixel (B).

b) Image part (2): an outward pixel is a condition that occurs when the next estimated-pixel (C) is outside the initial contour while the previous pixel (B) is inside the initial contour (A).

c) Image part (3): another outward pixel is a condition that also occurs when previous (B) and next estimated-pixel (C) are inside the initial contour (A), but the next estimated-pixed has a shorter displacement than the previous pixel.

d) Image part (4): an inward pixel is a condition that occurs when previous (B) and next estimated-pixel (C) are inside the initial contour (A), but the next estimated-pixed has a longer displacement than the previous pixel.

e) Image part (5): another inward pixel is a condition that also occurs when previous pixel (B) is outside the initial contour and the next estimated-pixel (C) are inside the initial contour (A).

f) Image part (6): an inward pixel is a condition that occurs when previous (B) and next estimated-pixel (C) are outside the initial contour (A), but the next estimated-pixed has a shorter displacement than the previous pixel. 


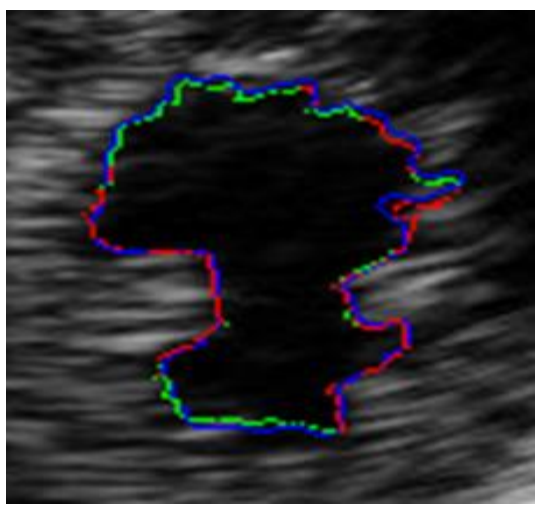

Figure 5. Result of Flow Direction Determination

Figure 5 shows the result of flow direction between two sequential images. Every direction is represented by different color. The blue pixels represent inward direction and the red pixels represent outward direction. The result showed that bit of pixels can be tracked correctly. However, the result can still be used as the next good features.

\subsection{Classification}

One cycle of heart started from diastole to systole is represented by 10 frames. So, there are nine changes between two consecutive images during one cycle. There are two features that will be extracted in one change that is flow direction $(\mathrm{F})$ and length of displacement $(\mathrm{L})$. Therefore, in one cycle of heart, there are 18 features that will be obtained and will be used as features in classification method. Artificial Neural Network is proposed for classifying the features become two classes that is normal and abnormal. Abnormal can be divided become three heart condition that is dyskinesia, akinesia, hypokinesia.

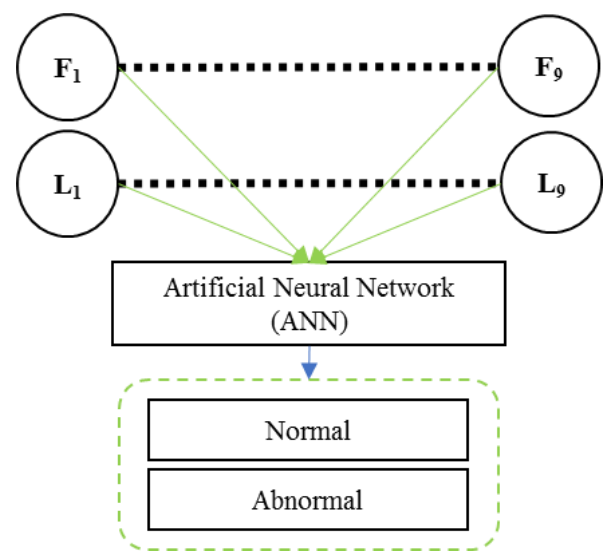

Figure 6. Proposed Classification Method

\section{RESULT AND DISCUSSION}

There are 11 sets of frames that successful to segment in this research. Those datasets are divided into two classes of normal and abnormal. Specifically, there are 5 normal datasets and 6 abnormal datasets. Proposed method in Figure 6 has been applied. Flow direction has been obtained and the statistic result of the feature described in Table 1.

Table 1. Statistic of Flow Direction

\begin{tabular}{ccc}
\hline Condition & Flow In (inward) & Flow Out (outward) \\
\hline Normal & $57 \%$ & $43 \%$ \\
Abnormal & $35 \%$ & $65 \%$ \\
\hline
\end{tabular}


Table 1 explain flow direction of normal and abnormal have different result. Normal class has the percentage value of inward flow higher than outward flow. Otherwise, abnormal class has the percentage value of inward flow lower than outward flow. These results correspond with the explanation in section 2 that the characteristic of normal heart is all of tissues moving inside. Table 1 shows difference percentage value between inward flow and outward flow in normal class is smaller than difference value in abnormal class. This is caused by there are still wrong determination in flow direction.

Classification is performed by using RapidMiner with few of classification methods. Table 2 shows the comparison result of every method. Percentage is obtained with Leave One Out (LOO) validation method. This validation method is commonly used to validate feature in medical computing case. It is observed that neural network with learning rate 0.01 has the highest performance with value $81.82 \%$. The second place is support vector machine with performance value is $72.73 \%$.

Table 2. Classification Results

\begin{tabular}{lc}
\hline \multicolumn{1}{c}{ Method } & Result $(\%)$ \\
\hline Nearest Neighbor $(\mathrm{N}=3)$ & $54.55 \%$ \\
Neural Network $(\mathrm{LR}=0.01)$ & $81.82 \%$ \\
Naïve Bayes & $63.64 \%$ \\
Support Vector Machine & $72.73 \%$ \\
\hline
\end{tabular}

\section{CONCLUSION}

Diagnose of cardiac motions by echocardiography expert is based on flow of direction and length of displacement of echocardiogram. Lucas-Kanade Optical Flow is applied in feature extraction to estimate good feature of all consecutive echocardiogram. Experiment in Figure 3 shows that Lucas-Kanade Optical Flow has good performance to estimate good feature in the next image. Flow direction can be determined almost precisely with proposed algorithm. Length of displacement can be calculated by using equation 8 . Flow Direction and length of displacement features has good enough performance by using neural network. However, optimization is needed to improve the result. This research proves those two features can be used to diagnose cardiac disease computationally.

\section{REFERENCES}

[1] J. G. Betts et al., "Anatomy and Physiology”. Houston: OpenStax, 2016.

[2] World Health Organization, "On World Heart Day WHO calls for accelerated action to prevent the world's leading global killer”, 2018. [Online]. Available: http://www.who.int/cardiovascular_diseases/en/. [Accessed: 06-Jul-2018].

[3] P. Torkashvand, H. Behnam, and Z. Sani, "Modified Optical Flow Technique for Cardiac Motions Analysis in Echocardiography Images," vol. 2, no. July, pp. 1-8, 2012.

[4] Maity, A. Pattanaik, S. Sagnika, and S. Pani, "A comparative study on approaches to speckle noise reduction in images," Proc. - 1st Int. Conf. Comput. Intell. Networks, CINE 2015, no. March, pp. 148-155, 2015.

[5] Y.-W. Song and S. S. Udpa, "A New Morphological Approach for Reducing Speckle Noise in Ultrasonic Images," pp. 1397-1400, 1997.

[6] N. Rajalakshmi, K. Narayanan, and P. Amudhavalli, "Wavelet-based weighted median filter for image denoising of MRI brain images," Indones. J. Electr. Eng. Comput. Sci., vol. 10, no. 1, pp. 201-206, 2018.

[7] S. A. Saleem and T. A. Razak, "An effective noise adaptive median filter for removing high density impulse noises in color images," Int. J. Electr. Comput. Eng., vol. 6, no. 2, pp. 611-620, 2016.

[8] O. Bobkova, V. V. Zyuzin, S. V. Porshnev, and V. V. Bobkov, "Experience of using of machine learning methods to identify the left ventricle region in echocardiographic records," 2016 IEEE 10th Int. Conf. Appl. Inf. Commun. Technol., pp. 1-5, 2016.

[9] M. S. Mansoory, M. Ashtiyani, and H. T. N., "Cardiac Motion Evaluation for Disease Diagnosis Using ICA Basis Neural Network,” 2009 Int. Assoc. Comput. Sci. Inf. Technol. - Spring Conf., no. 1, pp. 496-500, 2009.

[10] S. Mansor and J. A. Noble, "LOCAL WALL MOTION CLASSIFICATION OF STRESS ECHOCARDIOGRAPHY USING A HIDDEN MARKOV MODEL APPROACH Sarina Mansor, J Alison Noble," pp. 1295-1298, 2008.

[11] N. G. Pandian, D. J. Skorton, S. M. Collins, H. L. Falsetti, E. R. Burke, and R. E. Kerber, "Hetrogeneity of left ventricular segmental wall thickening and excursion in two dimensional echocardiograms of normal human subjects," vol. Am. J. Car, no. 51, pp. 1667-1673, 1983.

[12] C. Otto, R. Schwaegler, and R. Freema, "Textbook of Clinical Echocardiography”. 2013.

[13] R. Sigit, A. R. Barakbah, I. A. Sulistijono, and A. S. Aziz, "Automatic cardiac segmentation using triangle and optical flow,” Indones. J. Electr. Eng. Comput. Sci., vol. 8, no. 2, pp. 315-326, 2017. 